\title{
Produção de morangueiro cv. “Cegnidarem” sob túnel plástico
}

\author{
Cegnidarem strawberry yield in plastic tunnel system
}

\author{
Roberto Pedroso de Oliveira ${ }^{\mathrm{I}^{*}}$ Walkyria Bueno Scivittaro ${ }^{\mathrm{I}}$
}

\begin{abstract}
- NOTA -
\section{RESUMO}

O objetivo deste trabalho foi avaliar a produção de morangueiro "Cegnidarem", de dia neutro, comparandoa com a produção das cultivares "Aromas" $e$ "Diamante" nas condições climáticas da região Sul do Rio Grande do Sul. O experimento foi realizado em Pelotas, utilizando sistema de produção sob túnel baixo e irrigação por gotejamento. O transplantio das mudas foi realizado em julho de 2007. O delineamento experimental foi de blocos casualizados com parcelas subdivididas no tempo, com quatro repetições. As unidades experimentais foram constituídas por 30 plantas,

experimental design was a randomized complete block with split plot, with four replications. The experimental unit consisted of 30 plants. The number of fruits, yield and fresh fruit mass were analyzed weekly from October 2007 to January 2008. 'Cegnidarem' provided greater yield (613.9g of fruits per plant) than 'Aromas' (536.1g) and 'Diamante' (431.5g). 'Cegnidarem' also produced higher number of fruits per plant (39.2) than 'Aromas' (32.2) and 'Diamante' (22.6), but showed smaller average fruit fresh matter (15.3g) than 'Diamante' (18.5g). Concerning the yield performance, 'Cegnidarem' is another alternative to strawberry farmers of the Rio Grande do Sul South region.
\end{abstract} que foram avaliadas quanto ao número, à produção e à matéria fresca dos frutos, semanalmente, no período de outubro de 2007 a janeiro de 2008. A produção acumulada de frutos por planta da cv. "Cegnidarem" $(613,9 \mathrm{~g})$ é maior que a da "Aromas" (536,1g) e "Diamante" (431,5g). Além disso, o número de frutos produzidos por planta da "Cegnidarem" $(39,2)$ é maior que o da "Aromas" $(32,2)$ e o da "Diamante" $(22,6)$. No entanto, a matéria fresca média dos frutos da "Cegnidarem" $(15,3 g)$ é menor que a da "Diamante" $(18,5 g)$. Em função do desempenho produtivo obtido, a cv. "Cegnidarem" consiste em mais uma alternativa para os produtores de morango da região Sul do Rio Grande do Sul.

Palavras-chave: Fragaria x ananassa Duch., "Aromas", "Diamante", dia neutro.

\section{ABSTRACT}

The aim of this research was to evaluate the 'Cegnidarem' day-neutral strawberry yield, and to compare it to 'Aromas' and 'Diamante' under climatic conditions of the South region of Rio Grande do Sul state. The experiment was carried out in Pelotas, using tunnel system and dripping irrigation. The runners were transplanted in July 2007. The
Key words: Fragaria $x$ ananassa Duch., 'Aromas', 'Diamante', day neutral.

O morangueiro (Fragaria $\mathbf{x}$ ananassa Duch.) pertence à família das Rosaceas, sendo um híbrido resultante das espécies americanas $\boldsymbol{F}$. chiloensis, $\boldsymbol{F}$. virginiana e $\boldsymbol{F}$. ovalis, e da européia Fragaria vesca (RONQUE, 1998). No Brasil, a cultura encontra-se difundida em regiões de clima temperado e subtropical, onde se produz frutas para consumo in natura e para a industrialização (SANTOS, 2003).

A produção das plantas de morangueiro é afetada pela interação dos fatores temperatura e fotoperíodo, sendo as cultivares de dia neutro aquelas em que a indução floral ocorre de forma contínua sem interferência do fotoperíodo, sempre que as temperaturas sejam inferiores a $16^{\circ} \mathrm{C}$ (SANTOS, 2003). As cultivares de morangueiro mais utilizadas na região

IEmbrapa Clima Temperado, CP 403, 96001-970, Pelotas, RS, Brasil. E-mail: rpedroso@cpact.embrapa.br. *Autor para correspondência. 
Sul do Brasil provêm dos Estados Unidos (“Aromas”, "Camarosa”, "Camino Real”, "Diamante”, "Dover”, "Oso Grande”, "Sweet Charlie” e "Ventana”) e da Espanha ("Milsei-Tudla”) (OLIVEIRA et al., 2005). No Rio Grande do Sul, “Aromas” e "Diamante” são as cultivares de dia neutro mais utilizadas pelos agricultores.

A cv. “Aromas” foi lançada em 1997 pela Universidade da Califórnia, sendo descrita como muito produtiva. Ela possui hábito de crescimento ereto e os frutos são grandes, bastante firmes, com coloração vermelha acentuada, sabor agradável e qualidade excelente para consumo in natura e industrialização (SHASTA NURSERY, 2008; UNIVERSITY OF CALIFORNIA, 2008a). A cv. “Diamante” também foi lançada em 1997 pela Universidade da Califórnia. Ela possui hábito de crescimento ereto, sendo as plantas mais compactas que as da "Aromas”. Trata-se de uma cultivar medianamente produtiva, com frutos bem grandes, firmes e de coloração interna vermelha-clara, recomendados apenas para consumo in natura (UNIVERSITY OF CALIFORNIA, 2008b). A cultivar “Cegnidarem” foi obtida na Espanha em 1996 pela empresa Plantas de Navarra S.A. (PLANASA), sendo resultante do cruzamento das seleções nํ․ 86-037 e nํ․ 86-096. As plantas são vigorosas, com muitas folhas, que apresentam tamanho médio e tolerância ao oídio. A produção é precoce, uniforme e abundante durante todo o ciclo produtivo. Os frutos são de tamanho médio, formato cônico, epiderme vermelho-brilhante e de polpa vermelho-clara, firme e ligeiramente ácida (PLANASA, 2008).

Em razão da diversidade edafoclimática existente no país, o pequeno número de cultivares disponível tem sido um dos principais obstáculos ao desenvolvimento da cultura do morangueiro. Desse modo, a importância de serem incentivados programas nacionais de melhoramento genético e de serem introduzidas cultivares geradas em outros países.

O objetivo deste trabalho foi avaliar o desempenho produtivo da cultivar de morangueiro "Cegnidarem”, recentemente introduzida no Brasil, comparando-a com a das cultivares de dia neutro “Aromas” e "Diamante” nas condições climáticas da região Sul do Rio Grande do Sul.

O experimento foi realizado em Pelotas, RS (latitude 3146’19”, longitude 52²0’33”, altitude de $7 \mathrm{~m}$ acima do nível do mar e temperatura média anual de $17,6^{\circ} \mathrm{C}$ ). Foram avaliadas três cultivares de morangueiro (“Aromas”, “Diamante” e “Cegnidarem”), sendo utilizadas mudas procedentes da região da Patagônia, na Argentina. Os tratamentos foram distribuídos em delineamento experimental de blocos casualizados com parcelas subdivididas no tempo e quatro repetições, sendo as unidades experimentais constituídas por 30 plantas.

O transplantio das mudas foi realizado em 15 de julho de 2007, em canteiros de 1,2m de largura por $0,15 \mathrm{~m}$ de altura, espaçados entre si em $0,8 \mathrm{~m}$, em solo corrigido quanto à acidez para $\mathrm{pH}$ 6,0. O espaçamento entre linhas e entre plantas foi de $0,35 \mathrm{~m}$, sendo utilizadas três linhas por canteiro. Os canteiros foram revestidos com filme de polietileno preto com 30 micras de espessura. Utilizou-se o sistema de produção em túneis plásticos com 0,5m de altura na parte central. As plantas de morangueiro foram irrigadas por gotejamento, de acordo com a necessidade hídrica. O controle das plantas daninhas e a remoção de folhas secas ou com sintomas de doenças, de estolões e de frutos danificados ou com defeitos foram realizados manualmente. Semanalmente, as plantas foram fertirrigadas, com aplicações alternadas dos fertilizantes $\mathrm{KSC} 1^{\circledR}, \mathrm{KSC}^{\circledR}$ e nitrato de cálcio. O tratamento fitossanitário incluiu duas aplicações com iprodione e uma com azoxystrobin, para o controle de doenças fúngicas. Também foi realizada uma aplicação com abamectina, para o controle de ácaros, e foram utilizadas iscas para o controle de broca das frutas e de camundongos.

Durante o experimento, a umidade relativa média do ar e as temperaturas médias mínimas e máximas mensais foram de $82,2 \%, 6,0^{\circ} \mathrm{C}$ e $15,0^{\circ} \mathrm{C}$ para julho; $82,6 \%, 8,5^{\circ} \mathrm{C}$ e $16,7^{\circ} \mathrm{C}$ para agosto; $84,2 \%, 12,9^{\circ} \mathrm{C}$ e $21,7^{\circ} \mathrm{C}$ para setembro; $81,9 \%, 15,2^{\circ} \mathrm{C}$ e $23,7^{\circ} \mathrm{C}$ para outubro; $84,2 \%, 13,5^{\circ} \mathrm{C}$ e $24,7^{\circ} \mathrm{C}$ para novembro; e $81,6 \%, 17,2^{\circ} \mathrm{C}$ e $28,9^{\circ} \mathrm{C}$ para novembro, respectivamente.

Para a avaliação do experimento, considerou-se o período de colheita compreendido entre os meses de outubro de 2007 e janeiro de 2008, totalizando 16 avaliações totais. Em cada avaliação, foram determinados a matéria fresca e o número de frutos produzidos por unidade experimental. A matéria fresca média dos frutos foi calculada pela razão entre essas duas variáveis. Foram determinados também o número total e a produção acumulada de frutos, considerando-se o período integral de colheita. Em ambas as avaliações, consideraram-se apenas os frutos comercializáveis, descartando-se aqueles com defeitos e com massa inferior a 5g. Para a análise estatística, agruparam-se os dados a cada quatro semanas, perfazendo quatro períodos de avaliação. Os dados foram submetidos à análise de variância, comparandose as médias do fator cultivar pelo teste de Tukey $(\mathrm{P}<0,05)$ e do fator período de avaliação, por análise de regressão polinomial.

Ciência Rural, v.38, n.9, dez, 2008. 
Ao longo das 16 semanas de colheita, a "Cegnidarem” foi mais produtiva do que a "Aromas" e "Diamante”, sendo colhidos, respectivamente, 613,9; 536,1 e 431,5g de frutos comerciais por planta (Tabela 1). As produtividades obtidas foram satisfatórias, superando os 300-400g por planta considerada como a média do Rio Grande do Sul (PAGOT \& HOFFMANN, 2003). O maior potencial produtivo da "Cegnidarem” em relação às demais cultivares de dia neutro já havia sido relatado na Espanha (PLANASA, 2008). Os resultados apresentados neste trabalho referem-se às primeiras quantificações de produtividade da cultivar “Cegnidarem” no Brasil. Na tabela 1, verifica-se que a cultivar Aromas (536,1g por planta) foi $24,2 \%$ mais produtiva que a "Diamante" (431,5g). Este resultado concorda com os obtidos por SHAW (2004), que relata diferenças de produtividade de $25 \%$ a $30 \%$ em favor da “Aromas".

Ao longo do período de colheita, o número total de frutos produzidos por planta da cultivar “Cegnidarem” $(39,2)$ foi superior ao da “Aromas” $(32,2)$ e da "Diamante" $(22,6)$, demonstrando o potencial produtivo e a grande adaptabilidade da "Cegnidarem" às condições climáticas da região Sul do Rio Grande do Sul. No entanto, as plantas da cultivar "Cegnidarem” apresentaram menor matéria fresca média dos frutos $(15,3 g)$ em relação aos frutos da "Diamante" $(18,5 g)$ (Tabela 1). Estes resultados estão de acordo com a literatura, uma vez que os frutos da “Cegnidarem” são freqüentemente descritos como de tamanho médio (PLANASA, 2008), enquanto que os da "Diamante" são descritos como de tamanho grande (SHASTA NURSERY,2008).

De modo geral, as três cultivares apresentaram alta regularidade de produção, o que é uma característica muito valorizada pelos produtores. Nos quatro períodos de avaliação estudados, a cv. “Cegnidarem” foi mais produtiva, mas seus frutos eram menores que os frutos da "Aromas" e os frutos da “Aromas”, por sua vez, eram menores que os frutos da
"Diamante". Apenas no período da 5 a $8^{\mathbf{a}}$ semana de colheita não houve diferença, ao nível de 5\% de significância, quanto à produção de frutos das cultivares “Aromas" e "Diamante”.

Para as três cultivares de morangueiro estudadas, o efeito do período de colheita sobre a produção de frutos ajustou-se a modelos quadráticos de regressão (Figura 1). Segundo SANTOS (2003), a elevação das temperaturas médias promove redução na produção, mesmo nas cultivares de dia neutro, que independem do fotoperíodo para produzir. A maior produção de frutos comerciais por planta foi obtida na $12^{\mathrm{a}}$ semana de colheita para as cultivares "Cegnidarem” $(198,8 g)$ e “Aromas” $(177,2 g)$ e na 11ª semana para a "Diamante” (159,4g). Após a 13ª semana de colheita, a produção de frutos comerciais das três cultivares diminuiu.

Mesmo com o aumento das temperaturas médias mínimas e máximas mensais ao longo do período de avaliação, as três cultivares estudadas mantiveram o nível de floração, porém, ocorreu aumento do número de frutos que não eram comercializáveis. Mesmo assim, o efeito do período de avaliação sobre o número de frutos comerciais produzidos ajustou-se ao modelo linear para a cultivar "Cegnidarem”, o que indica aumento crescente no número de frutos produzidos ao longo das 16 semanas de colheita.

A variação na matéria fresca média dos frutos ao longo do período de colheita, para as três cultivares avaliadas, ajustou-se a um modelo quadrático (Figura 1). A maior matéria fresca de fruto foi obtida na $10^{\mathrm{a}}$ semana de colheita para as cultivares "Diamante" $(23,1 \mathrm{~g})$ e "Cegnidarem" $(19,2 \mathrm{~g})$ e na 9 a semana para a “Aromas” (19,9g). Os resultados evidenciam o potencial das cultivares estudadas, especialmente da "Diamante", para a produção de frutos de tamanho extra (>14g). Na figura 1, observa-se que os frutos da cultivar “Diamante” apresentaram maior massa média durante todo o período de colheita avaliado, enquanto

Tabela 1 - Produção acumulada, número e massa fresca média de três cultivares de morangueiro (Fragaria x ananassa Duch.). Pelotas, RS. Outubro de 2007 a janeiro de 2008.

\begin{tabular}{lccc}
\hline Cultivar & Produção acumulada $\left(\mathrm{g} \mathrm{planta}^{-1}\right)$ & Número de frutos (frutos planta $\left.^{-1}\right)$ & Massa fresca média $\left(\mathrm{g}\right.$ fruto $\left.^{-1}\right)$ \\
\hline "Cegnidarem” & $613,9 \mathrm{a}$ & $39,2 \mathrm{a}$ & $15,3 \mathrm{~b}$ \\
"Aromas” & $536,1 \mathrm{~b}$ & $32,2 \mathrm{~b}$ & $16,4 \mathrm{ab}$ \\
"Diamante” & $431,5 \mathrm{c}$ & $22,6 \mathrm{c}$ & $18,5 \mathrm{a}$ \\
& & & 6,2 \\
\hline
\end{tabular}

Médias seguidas de mesma letra, nas colunas, não diferem significativamente entre si pelo teste de Tukey a 5\%. 


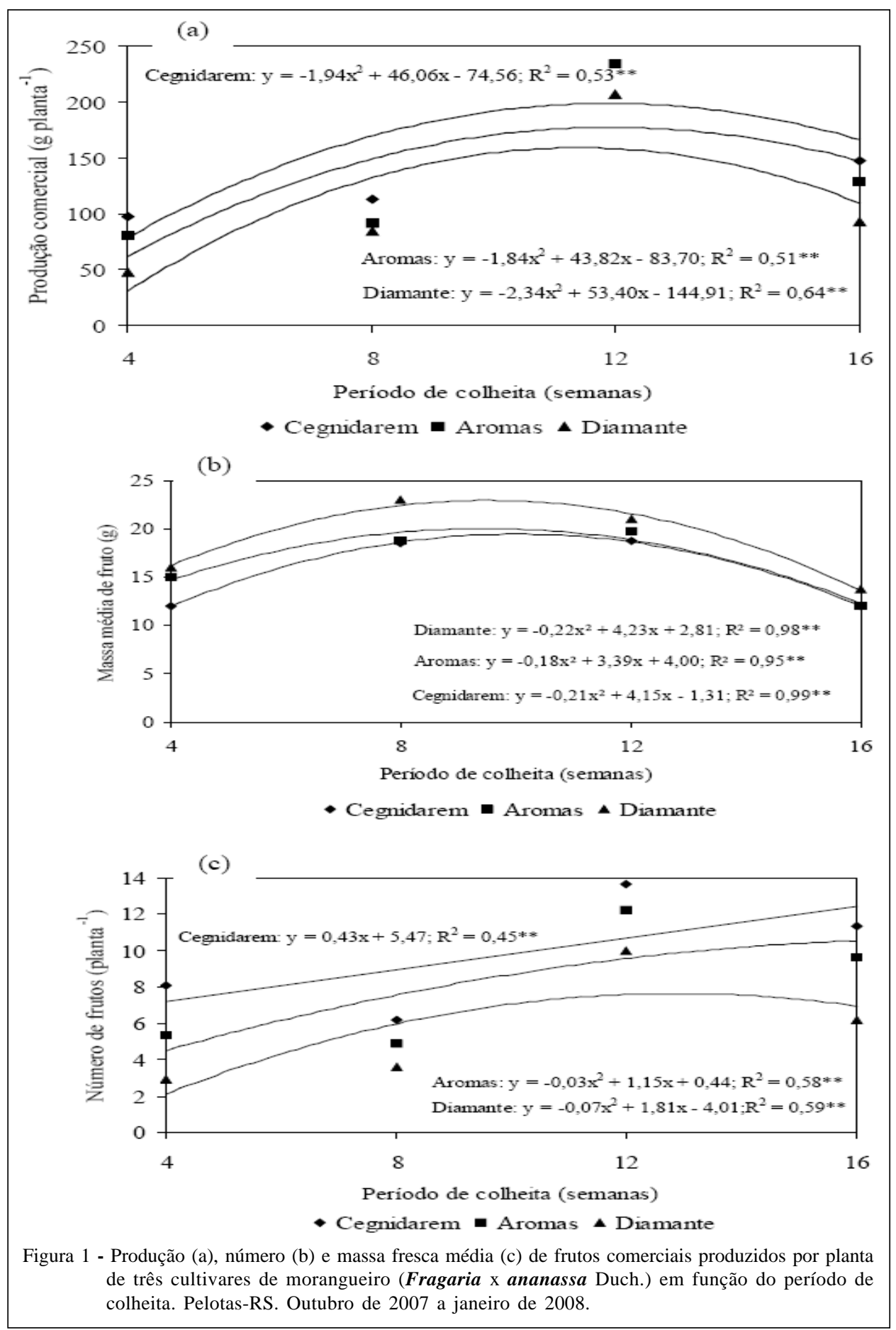

que os da "Cegnidarem” apresentaram os menores valores médios.

Pelos resultados obtidos, pode-se concluir que a "Cegnidarem” é mais produtiva que a "Aromas" e a "Diamante", produzindo maior número de frutos, embora estes apresentem menor matéria fresca que a “Diamante”. Desse modo, “Cegnidarem” consiste em mais uma alternativa para os produtores de morango da região Sul do Rio Grande do Sul.

\section{AGRADECIMENTOS}

À Fundação de Apoio à Pesquisa do Estado do Rio Grande do Sul (FAPERGS) e ao Conselho Nacional de

Ciência Rural, v.38, n.9, dez, 2008. 
Desenvolvimento Científico e Tecnológico (CNPq), pelo apoio financeiro e pela concessão de bolsa de estudo.

\section{REFERÊNCIAS}

OLIVEIRA, R.P. et al. Mudas certificadas de morangueiro: maior produção e melhor qualidade da fruta. A Lavoura, Rio de Janeiro, v.108, p.35-38, 2005.

PAGOT, E.; HOFFMANN, A. Produção de pequenas frutas. In: SEMINÁRIO BRASILEIRO SOBRE PEQUENAS FRUTAS, 2003, Vacaria, RS. Anais... Bento Gonçalves: Embrapa Uva e Vinho, 2003. p.9-17. (Embrapa Uva e Vinho. Documentos, 37).

PLANASA. Cegnidarem. Capturado em 09 jan. 2008. Online. Disponível na Internet: http://planasa.com/product/ Fresa/cegnida/cegnida.htm

RONQUE, E.R.V. Cultura do morangueiro; revisão e prática. Curitiba: Emater, 1998. 206p.
SANTOS, A.M. Cultivares. In: SANTOS, A.M.; MEDEIROS, A.R.M. Morango: produção. Brasília: Embrapa Informação Tecnológica, 2003. p.24-30. (Embrapa Informação Tecnológica. Frutas do Brasil, 40).

SHASTA NURSERY. Variety list. Capturado em 09 jan. 2008 . Online. Disponível na Internet: http://www.rootstock.com/ variety.html

SHAW, D.V. Strawberry production systems, breeding and cultivars in California. In: RASEIRA, M.C.B. et al. Morango. Pelotas: Embrapa Clima Temperado, 2004. p.16-21. (Embrapa Clima Temperado. Documentos, 124).

UNIVERSITY OF CALIFORNIA. Aromas strawberry cultivar. Capturado em 09 jan. 2008a. Online. Disponível na Internet: http://www.ucop.edu/ott/strawberry/ Aromascultivar.htm

UNIVERSITY OF CALIFORNIA. Diamante strawberry cultivar. Capturado em 09 jan. 2008b. Online. Disponível na Internet: http://www.ucop.edu/ott/strawberry/ Diamantecultivar.htm 